\title{
Sindicación de contenidos en un portal de revistas: Temaria
}

\author{
Por Josep Manuel Rodríguez Gairín, Jorge Franganillo, Ernest Abadal, Assumpció Estivill y Jesús Gascón
}

Resumen: Se describe la sindicación de contenidos y sus aplicaciones en bibliotecas, centros de información y editoriales, y se destaca como una oportunidad en un momento en que las iniciativas en este terreno son todavía escasas. Se explica cómo la sindicación aplicada a Temaria, un portal de revistas españolas de biblioteconomía y documentación, facilita, entre otras cosas, el seguimiento de un autor o de un tema.

Palabras clave: Sindicación de contenidos, RSS, Difusión selectiva de la información, Temaria.

\section{Title: Content syndication in a journal portal: Temaria}

Abstract: This paper describes content syndication and its applications for libraries, information centres and publishers, and highlights it as a new opportunity considering the scant developments in this field. Further, it explains how syndication, as applied to Temaria, a portal of Spanish journals on library and information science, makes it easier to track an author or to keep up-to-date on specific subjects, amongst other possibilities.

Keywords: Content syndication, RSS, Selective information dissemination, Temaria.

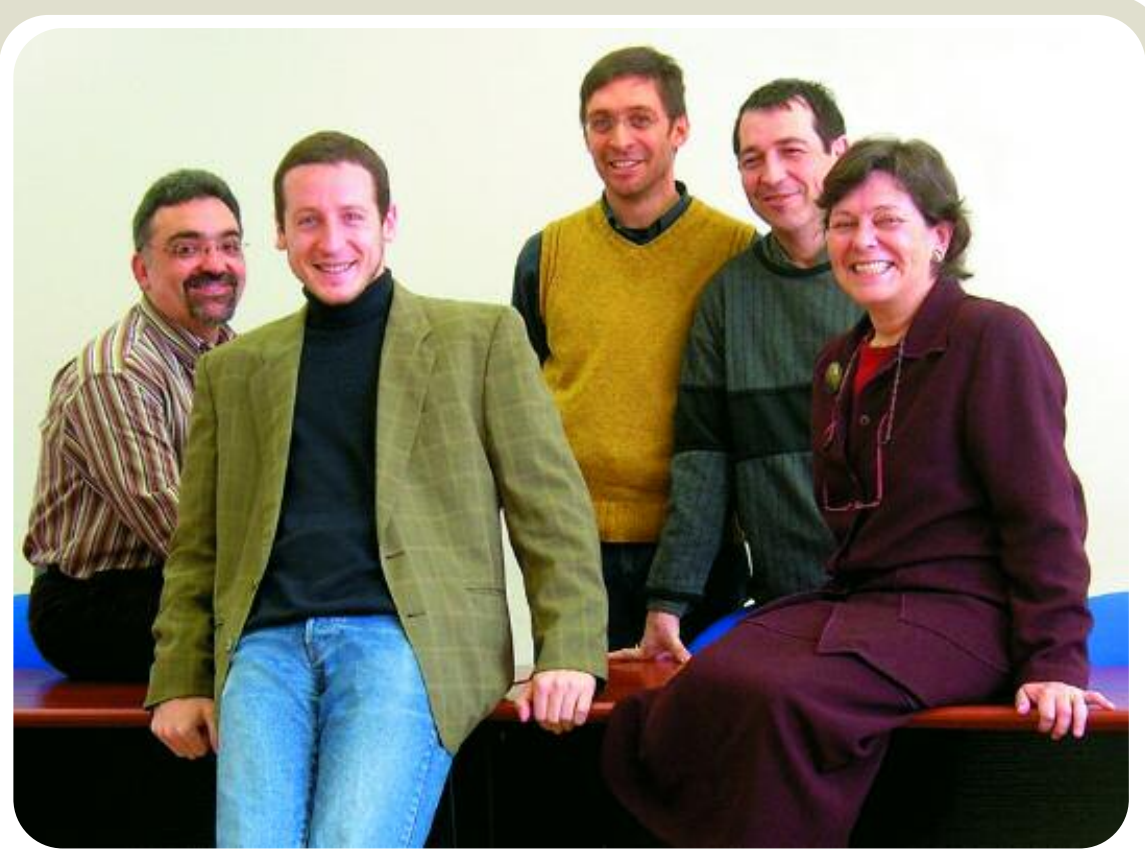

Josep Manuel Rodríguez Gairín es profesor de edición electrónica, lenguajes de etiquetado y sistemas de gestión de contenidos basados en web. Se ocupa de los aspectos tecnológicos y de la aplicación que gestiona Temaria.

Jorge Franganillo, profesor de edición electrónica y bases de datos, es autor de varios textos sobre recuperación de información. Ha colaborado en el diseño de recursos electrónicos diversos y ha concebido la interfaz y el diseño gráfico de Temaria.

Ernest Abadal, profesor de tecnologías de la información, es autor de diversos textos sobre bases de datos documentales y sobre publicaciones digitales. Es coordinador de contenidos del portal Temaria.

Jesús Gascón, profesor de catalogación y lenguajes documentales, es autor de algunos textos sobre estas materias. Es responsable de los criterios de aplicación de descriptores en Temaria, así como del mantenimiento del tesauro utilizado.

Assumpció Estivill, profesora de catalogación y organización de recursos electrónicos, es autora de textos sobre estos temas; recientemente ha coordinado el Libro blanco del tífulo de grado en información y documentación. Es responsable de metadatos del portal Temaria.

Rodríguez, Gairín, Josep Manuel; Franganillo, Jorge; Abadal, Ernest; Estivill, Assumpció; Gascón, Jesús. «Sindicación de contenidos en un portal de revistas: Temaria». En: El profesional de la información, 2006, mayo-junio, v. 15, n. 3, pp. 214-221.

\section{Introducción}

LA APARICIÓN DE BLOGS (diarios interactivos también conocidos como weblogs o bitácoras) y la proliferación de medios de comunicación y agencias de noticias, han dispersado los canales de acceso a la información de actualidad.

Como consecuencia, los usuarios tropiezan con dificultades para seguir con atención, sin perderse, la aparición de nuevos contenidos. Esta situación les obliga a contar con dispositivos que los localicen, integren y clasifiquen en un punto común. De esta tarea se ocupan los programas agregadores, que se sustentan en una tecnología basada en $\mathrm{xml}$, un lenguaje pensado para poder definir todo tipo de contenidos de una forma estándar y fácilmente comprensible ya sea por cualquier persona o programa de ordenador preparado al efecto.

La sindicación es el proceso mediante el cual un productor o un distribuidor de contenidos en internet los proporciona a un suscriptor, o a una red de suscriptores. Éstos pueden utilizar un agregador de noticias (lector de fuentes de sindicación) para suscribirse a diversos canales y recibir notificaciones del 


\section{चinfonomia IARED DE INWOVADORES}

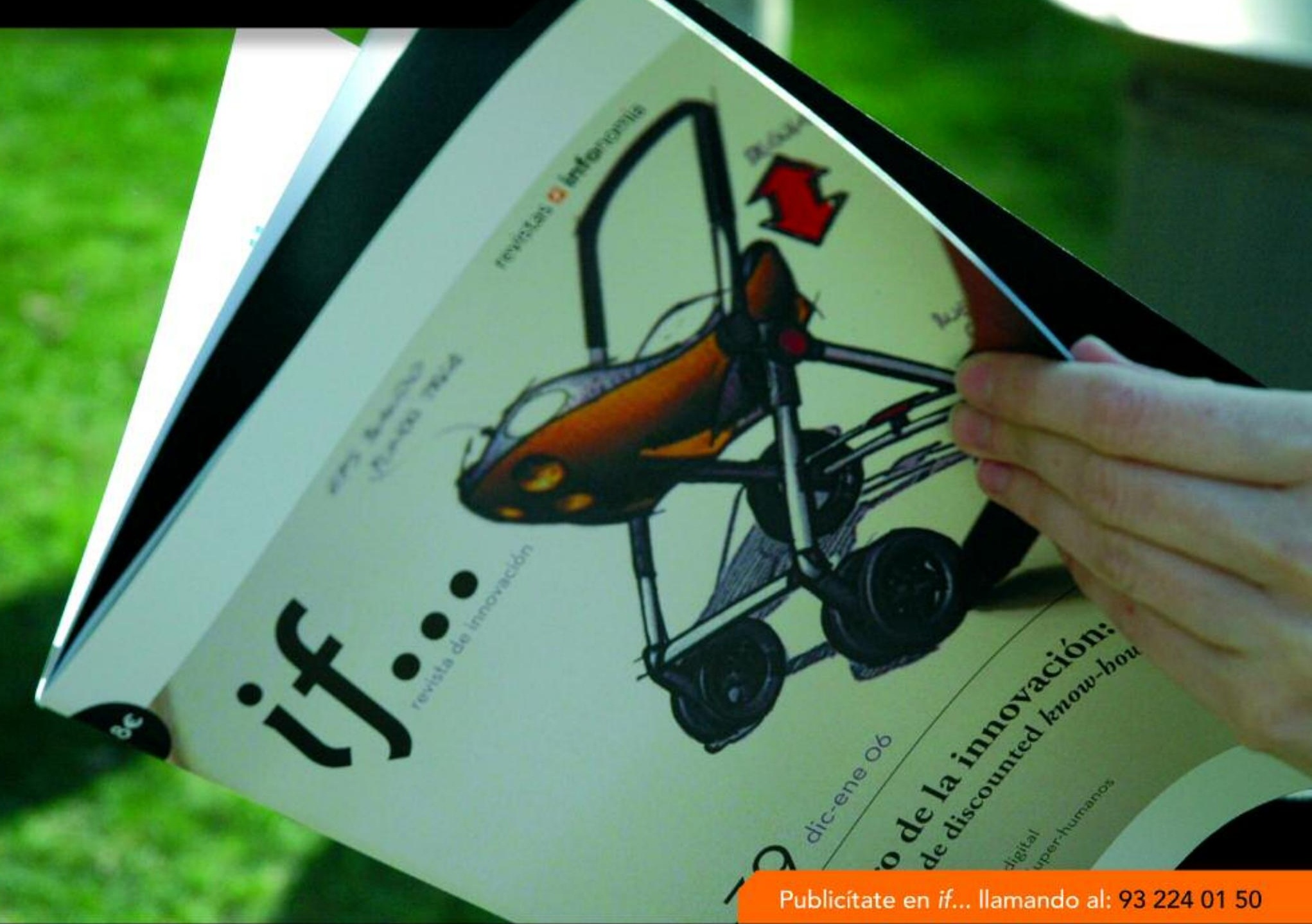

\section{LA REVISTA if..}

La revista mensual independiente de referencia sobre innovación. Llega a los profesionales y directivos más inquietos de las empresas más innovadoras.

Subscripción corporativa: www.infonomia.com/corp Subscripción individual: www.infonomia.com/revistaif

\section{LA RED DE INNOVADORES}

Los mejores profesionales, dispuestos a compartir sus experiencias. Pertenecer a nuestra red tiene sus ventajas.

Busca tu perfil: www.infonomia.com/directorio 
ámbito que les interese. De esta forma reciben los titulares, que conducen al texto completo de las noticias y a otras informaciones de actualidad. Si disponen de una sede web pueden integrar allí esos contenidos que obtienen automáticamente (Silva, 2004), para proporcionar sin necesidad de incorporar tecnología adicional una información con valor añadido capaz de fidelizar a los lectores. Así, el sitio web no sólo ofrece un producto o un servicio, sino que también vela por suministrar información útil y actual.

\section{«Mediante la sindicación, un productor o un distribuidor de contenidos proporciona información digital a un suscriptor, o a una red de suscriptores»}

En definitiva, la sindicación de contenidos es un buen ejemplo de la conocida difusión selectiva de información (DSI), un servicio que viene descrito en todos los manuales de biblioteconomía y documentación desde hace más de treinta años, algunas de cuyas primeras aplicaciones se dieron en los medios de comunicación, especialmente en prensa y agencias de noticias. Por otro lado, la consolidación de los blogs ha supuesto otro punto de interés, ya que la práctica totalidad de estos medios de publicación sindica sus fondos. En estos dos ejemplos de fuentes informativas se observa, pues, un uso intensivo de la sindicación, un mecanismo que, de hecho, resulta oportuno en cualquier sede web. Además, se distingue como uno de los éxitos destacables del lenguaje $\mathrm{xml}$, porque ha democratizado la distribución de noticias y porque convierte a cualquier persona en un proveedor potencial de contenidos.

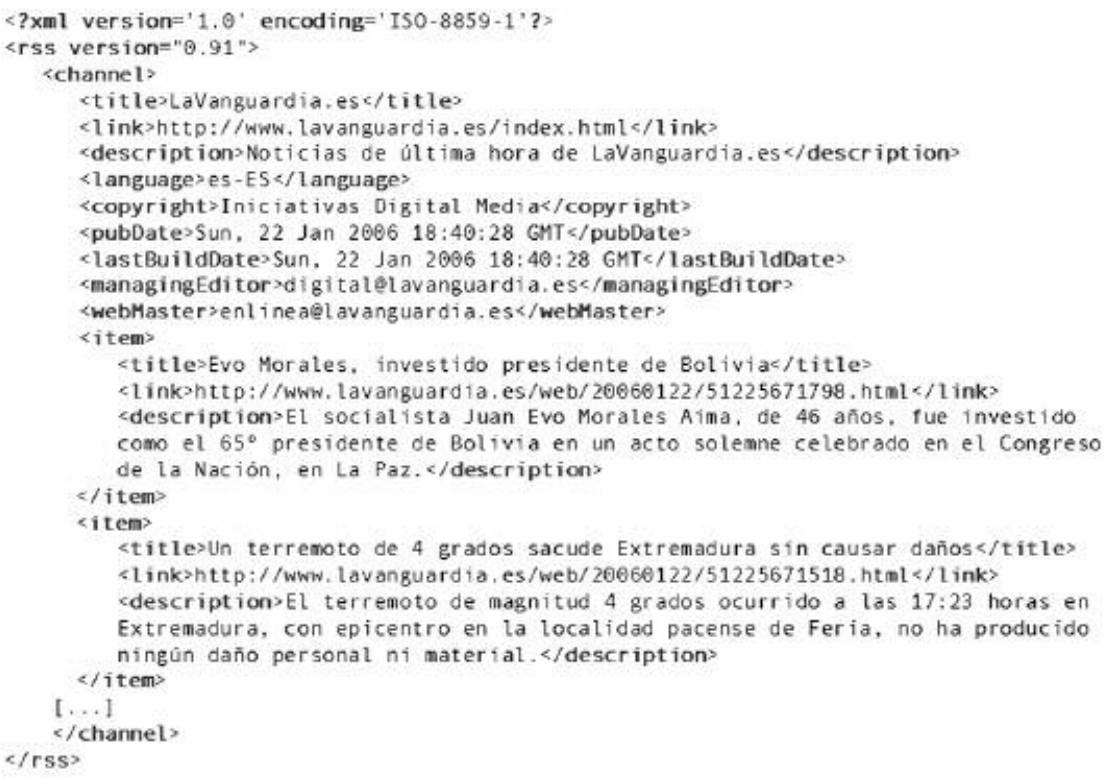

Figura 1. Fuente RSS 0.91 del periódico La vanguardia digital.

Abundan los textos que se centran en la sindicación y en el formato $R S S$, pero son pocos los que se han publicado en relación con la biblioteconomía y documentación. Entre éstos cabe destacar los artículos de Moffat (2003) y Wusterman (2004), ambos dedicados a describir aspectos técnicos y aplicaciones en este contexto. Tres trabajos sobresalen en España. El primero es de carácter más bien teórico: Portela (2001) se hace eco de las nuevas oportunidades que se presentan para los profesionales de la información y la documentación en el entorno digital; en un apartado se refiere explícitamente a la sindicación (juntamente con los portales y la arquitectura de la información). Más técnico, el artículo de Serrano (2004) describe el formato RSS, el funcionamiento de los agregadores de noticias y su aplicación a Google. El más reciente es el de Franganillo y Catalán (2005), que describe los antecedentes, la evolución y las aplicaciones de las bitácoras y la sindicación de contenidos, incluyendo consideraciones técnicas y ejemplos.

A continuación se presenta y se comenta la implantación de RSS que se ha llevado a cabo en Temaria, un portal que indiza artículos de revistas españolas de biblioteconomía y documentación y que facilita al usuario la actualización de informaciones de un autor o sobre una materia. Pero antes se repasan dos conceptos clave: sindicación y RSS.

\section{Sindicación de contenidos y RSS}

En general, la redifusión de contenidos se lleva a cabo a través de dos formatos: RSS y Atom ${ }^{1}$. Google adoptó Atom en 2004 y le dio notoriedad, pero actualmente impulsa los dos. $R S S$ es anterior y está basado en rdf, el estándar de metadatos del W3C. Apareció en 1997 y su elaboración fue tarea de varias organizaciones que trabajaron de forma dividida (Pilgrim, 2002), circunstancia que explica que hoy convivan siete versiones de $R S S$, diferentes entre sí: las siglas RSS representan Rdf Site Summary en la versión 0.90, Rich Site Summary en las 0.91-0.94 y 1.0, y Really Simple Syndication en la 2.0. El RSS 2.0 es el estándar que se utiliza en Temaria.

Las fuentes RSS (figura 1) son documentos xml destinados a la distribución de noticias breves. Su estructura es simple. Consta de un nodo primario <channel $>$ con cuatro subnodos obligatorios: <title> 
(el título del conjunto de noticias o canal), <link> (url del fichero), $<$ description $>$ (una descripción del canal), e <item $>$ (el texto de las noticias). Este último nodo es repetible y se dispone un <item $>$ para cada noticia, y cada una de ellas tiene a su vez los subnodos <title $>$, $<$ link $>\mathrm{y}<$ description $>$. Tanto $<$ channel> como <item $>$ pueden tener subnodos opcionales, de los que se puede consultar una enumeración completa en la especificación $R S S 2.0$.

http://blogs.law.harvard.edu/tech/rss

Los blogs y las sedes web que distribuyen noticias generan estos documentos con el objetivo de que

\begin{tabular}{|c|c|c|}
\hline \multicolumn{3}{|c|}{ Ámbito editorial } \\
\hline \multirow{5}{*}{$\begin{array}{l}\text { Distribución } \\
\text { de sumarios } \\
\text { de revistas }\end{array}$} & Ariadne & http://www.ariadne.ac.uk \\
\hline & \begin{tabular}{|l|}
$D$-lib \\
magazine \\
\end{tabular} & http://dlib.org \\
\hline & $\begin{array}{l}\begin{array}{l}\text { Information } \\
\text { research }\end{array} \\
\end{array}$ & http://informationr.net/ir \\
\hline & \begin{tabular}{|l|} 
Institute of \\
Electrical and \\
Electronics \\
Engineers \\
\end{tabular} & http://www.computer.org/portal/cms_docs_cs/csdl/jsp/rss \\
\hline & \begin{tabular}{|l|} 
Oxford \\
University \\
Press \\
\end{tabular} & http://www.oxfordjournals.org/our_journals \\
\hline \multirow{3}{*}{$\begin{array}{l}\text { Aviso de } \\
\text { nuevas } \\
\text { publicaciones } \\
\text { Reseñas }\end{array}$} & $\begin{array}{l}\text { Harvard } \\
\text { University } \\
\text { Press } \\
\end{array}$ & http://www.hup.harvard.edu/rss/what_is_rss.html \\
\hline & Poliedro & http://poliedro.com \\
\hline & Routledge & http://brunner-routledge.co.uk \\
\hline
\end{tabular}

Bases de datos bibliográficas

\begin{tabular}{|l|l|l|}
\hline $\begin{array}{l}\text { Distribución } \\
\text { de alertas }\end{array}$ & PubMed & http://pubmed.org \\
\hline
\end{tabular}

\begin{tabular}{|l|l|}
\hline Bitácoras \\
\hline $\begin{array}{l}\text { Difusión de } \\
\text { nuevos } \\
\text { contenidos }\end{array}$ & La práctica totalidad de estos recursos sindica sus contenidos. \\
\hline
\end{tabular}

\begin{tabular}{|l|l|l|}
\hline \multicolumn{2}{|l|}{ Webs de centros de información } \\
\hline \multirow{2}{*}{$\begin{array}{l}\text { Informe de } \\
\text { nuevos } \\
\text { fondos, } \\
\text { servicios y } \\
\text { actividades }\end{array}$} & $\begin{array}{l}\text { Australian } \\
\text { National } \\
\text { University } \\
\text { Library }\end{array}$ & http://anulib.anu.edu.au/epubs/innopacnewbooksrss.html \\
\cline { 2 - 3 } & Bibliotècnica & http://bibliotecnica.upc.es \\
\cline { 2 - 3 } & Curtin Library & http://library.curtin.edu.au/research/rss \\
\hline
\end{tabular}

\begin{tabular}{|l|l|l|}
\hline \multicolumn{2}{|l|}{ Portales de revistas } \\
\hline $\begin{array}{l}\text { Difusión de } \\
\text { los } \\
\text { contenidos } \\
\text { indizados, a } \\
\text { modo de } \\
\text { alertas y } \\
\text { sumarios }\end{array}$ & Temaria & http://temaria.net \\
\hline
\end{tabular}

Tabla. Aplicaciones de la sindicación en biblioteconomía y documentación http://blogbridge.com

http://rssreader.com

http://rssowl.org

http://bloglines.com

http://google.com/reader

Gracias a ellos el suscriptor se beneficia de la sindicación porque ya no se verá obligado a recorrer páginas que tal vez no ofrezcan ninguna novedad, y así ahorra tiempo y esfuerzo. Los programas se conectan a internet automáticamente, buscan contenidos nuevos, y si los hay se lo notifican al usuario; le presentan entonces una lista de titulares, en forma de enlaces hipertextuales, con los resúmenes correspondientes. Al activar los vínculos oportunos se abre el navegador presentando la información que se le haya pedido y el interesado podrá consultar la que desee. Los agregadores, por su propia naturaleza, ofrecen además otras ventajas: confeccionan un noticiario hecho a medida, libran del correo basura y garantizan el anonimato al usuario, que no tendrá más noticias de un editor una vez que haya anulado la suscripción.

\section{Aplicaciones en biblioteconomía y documentación}

Aunque todavía escasas, son diversas las utilidades de la sindicación en este contexto. Cabe destacar los ejemplos de las revistas digitales, bases de datos, portales de revistas, blogs y sedes web de centros de información (ver tabla); de cada uno hay muestras varias, la mayoría foráneas, pues aún son pocos los casos de aplicación en España. En este ámbito, tanto Wusteman (2004) como Franganillo y Catalán (2005) ofrecen ejemplos concretos.

El hecho de que Rdf Site Summary sea uno de los desgloses de las siglas $R S S$ explica que las editoriales conciban este formato como un mecanismo de uso frecuente para la distribución de sumarios de revistas. 
periències), Núria Jornet (Textos

licència de Creative Commons * RSS

Internet

Figura 2. Botón de sindicación junto al sumario de BiD, revista indizada en Temaria

Muchos editores han utilizado el correo electrónico como el canal habitual para mandar alertas y tablas de contenido, pero en estos últimos años el sector ha comenzado a usar los mecanismos de sindicación. De ello son ejemplos la editorial $O x f o r d$ University Press y la biblioteca digital del Institute of Electrical and Electronics Engineers.

\section{«La sindicación ahorra tiempo y molestias al usuario porque le libra de recorrer páginas que quizá no ofrezcan novedades»}

En enero de 2005 la Universidad de Iowa compiló un directorio de revistas que tienen prestaciones de sindicación ${ }^{3}$; allí se citan cinco títulos del campo de la biblioteconomía y documentación, entre los que cabe destacar Ariadne, D-lib magazine e Information research. Pero la sindicación en el ámbito editorial no está confinada a sumarios de revistas: cada vez son más numerosas las empresas que informan mediante RSS de los libros que incorporan al catálogo. Son ejemplos las editoriales Routledge, Harvard University Press y Poliedro.

Otro entorno que ofrece muchas posibilidades, aunque aún por desarrollar, es el de las bases de datos bibliográficas y los portales de revistas. Sirva de ejemplo la interfaz de consulta de PubMed, que permite crear perfiles de consulta por medio de un canal $R S S$ y reformula así los servicios de alerta bibliográfica.

En la blogosfera sobre biblioteconomía y documentación, blogs
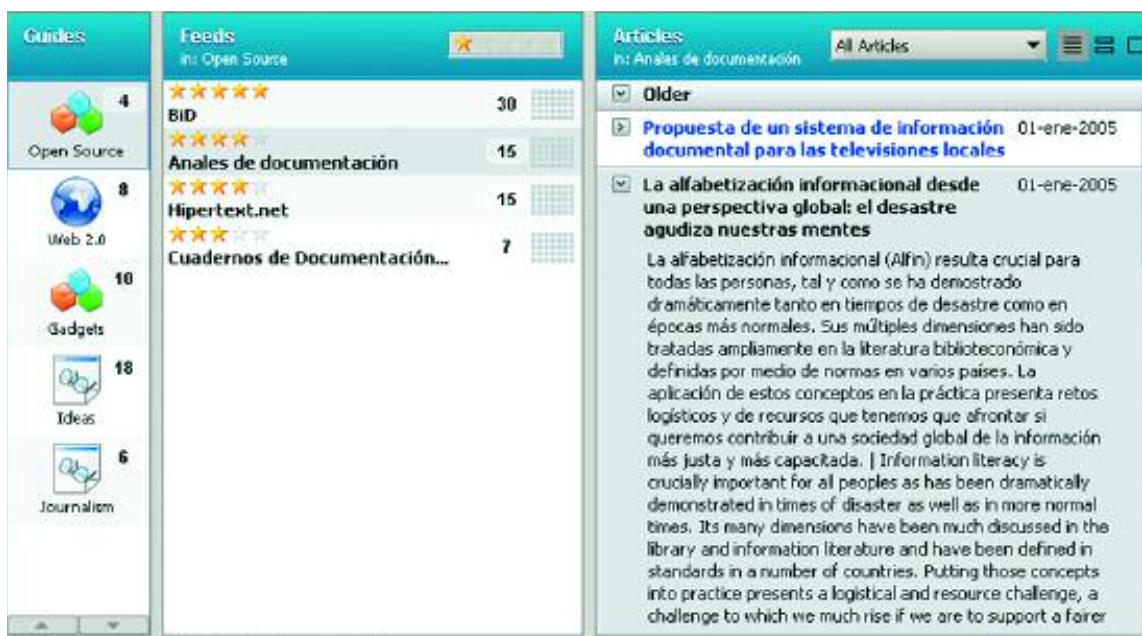

Figura 3. A través de varios canales, el lector BlogBridge obtiene los sumarios de los últimos números de algunas revistas indizadas en Temaria

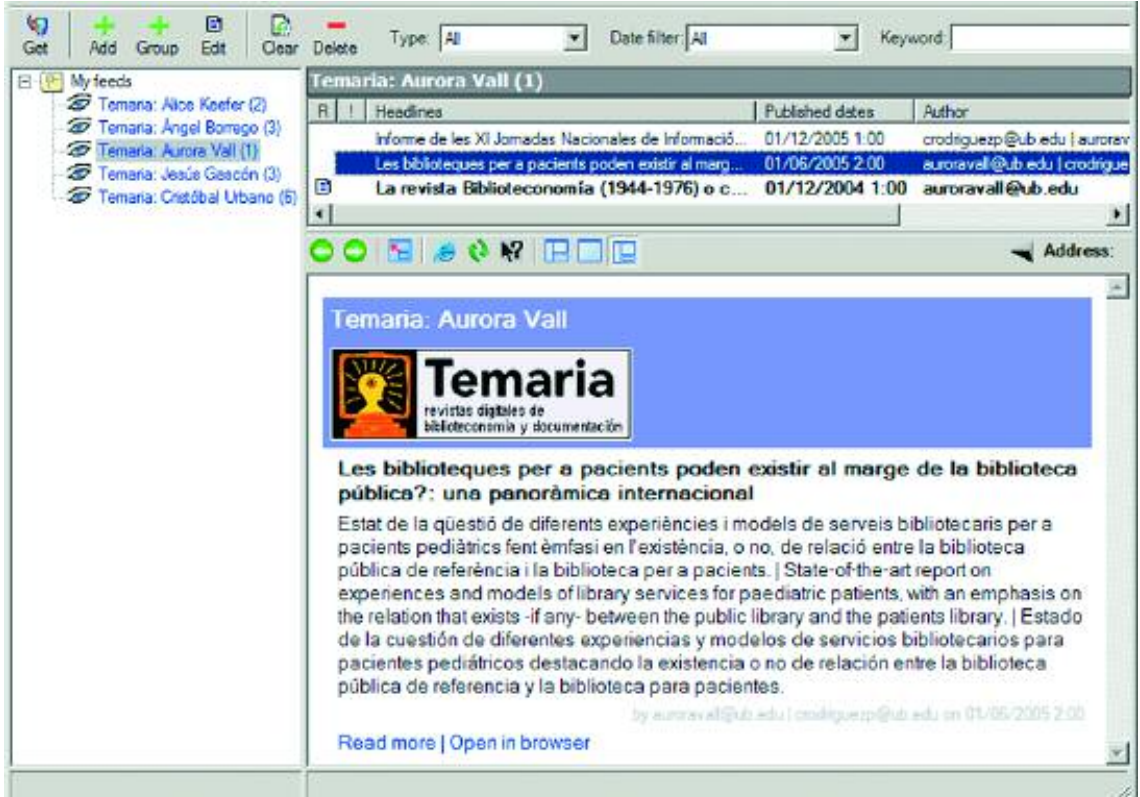

Figura 4. Alerta creada con RssReader para tener noticia de nuevos trabajos firmados por Aurora Vall

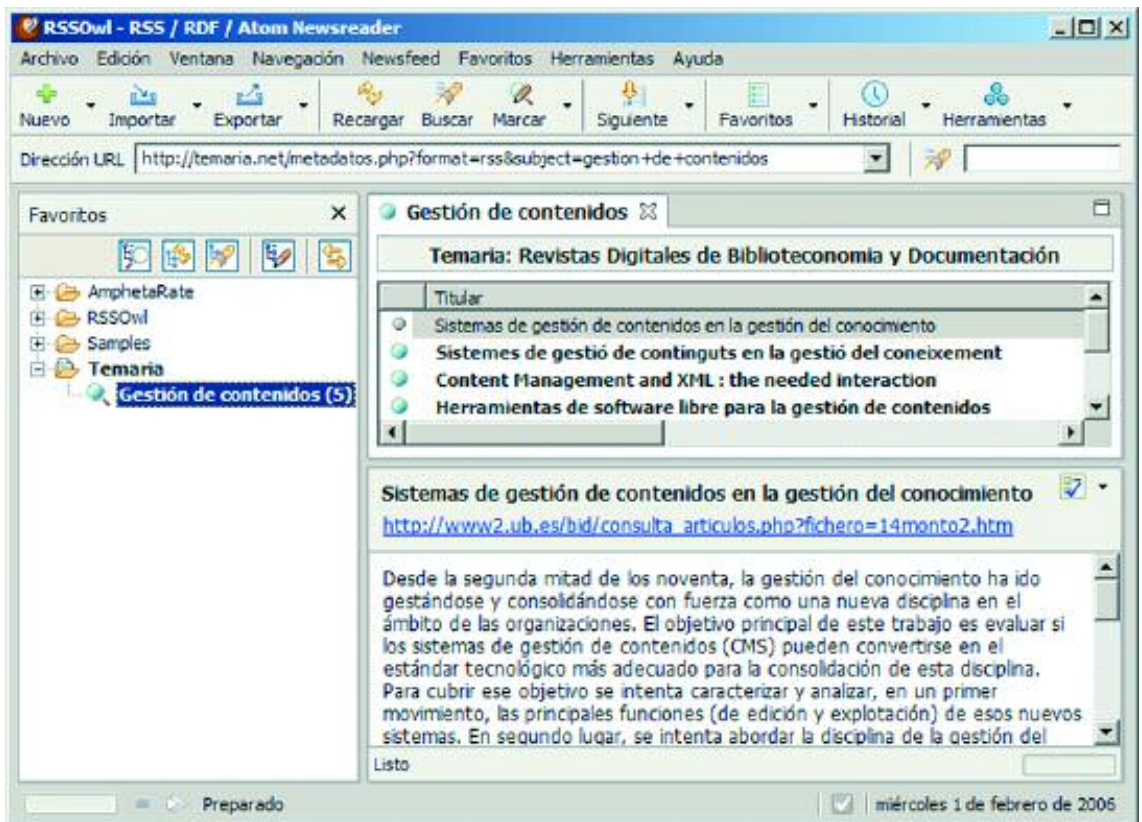

Figura 5. Seguimiento del tema "gestión de contenidos" mediante el lector RSSOwl 


\section{Años de experiencia}

Esto es lo que EBSCO ofrece.

Nuestro personal gestiona sus suscripciones a revistas electrónicas individuales o incluidas en ' paquetes de revistas, suscripciones, en papel y bases de datoś.

La Lista A-Z (A-to-Z) agrupa todos: sus recursos electrónicos en una misma lista, para que sus usuariọs localicen de manera rápida los títulos disponibles y accedan fácilmente a los contenidos. Además, la lista A-to-Z alimenta ál servidor de enlaces LinkSource ${ }^{\text {, }}$, que utilizando la norma Open'URL enlaza todos sus recursos de forma compacta e inteligente.

Experiencia, servicio, contenido, soluciones.

Hablemos hoy de sus necesidades.

CUSTOMERFOCUSEDCONTENTDRIVEN

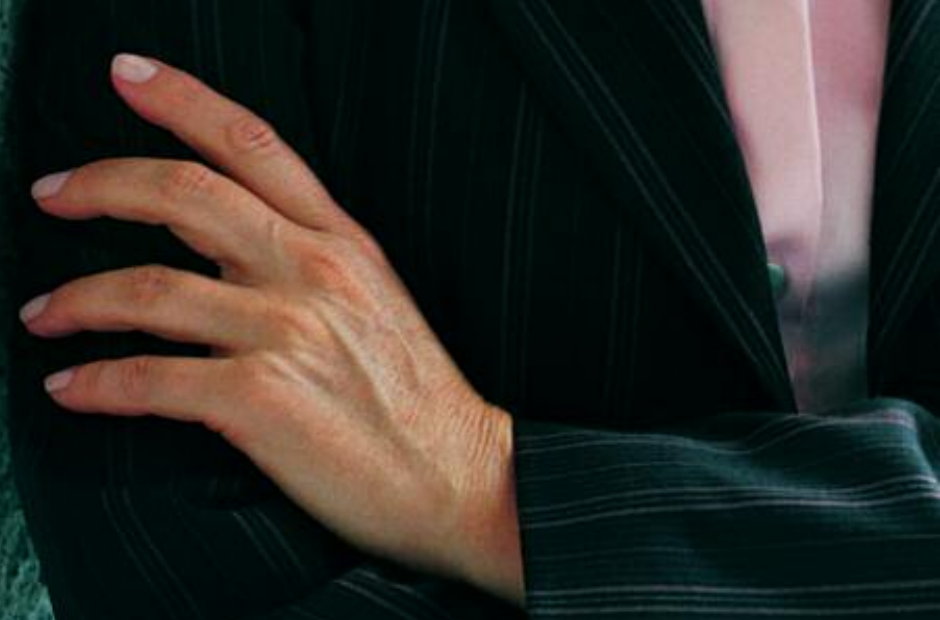
www.ebsco.com
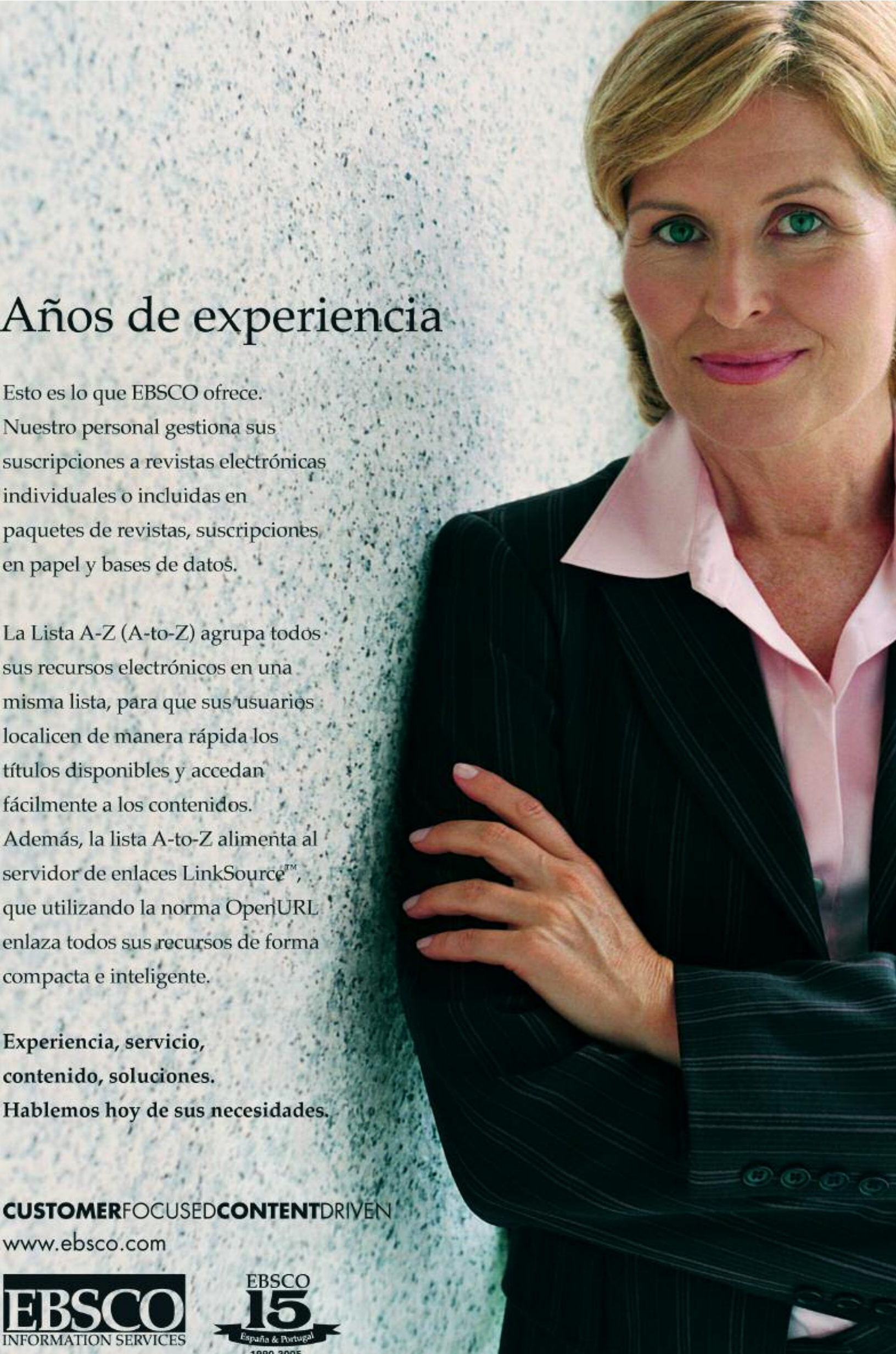
como El documentalista enredado o Deakialli DocuMental demuestran interés en averiguar cómo la sindicación puede contribuir a que los servicios de los centros de información sumen valor, y se hacen eco de algunas aplicaciones en ese sentido. La sindicación puede entonces aplicarse, con fines diversos, a la sede web de bibliotecas, archivos o centros de documentación. Algunas bibliotecas, como la Curtin Library y la Australian National University Library, han incorporado esta prestación como medio para dar noticia de sus últimas adquisiciones. Por su parte, Bibliotècnica, la biblioteca digital de la Universitat Politècnica de Catalunya, difunde así novedades sobre sus servicios y sobre las actividades que promueve.

http://documentalistaenredado.net http://deakialli.bitacoras.com

\section{Aplicación en Temaria}

Este portal de revistas permite acceder al texto completo de artículos de publicaciones españolas de información y documentación. La búsqueda es multilingüe, facilita el acceso por materias y se lleva a cabo por medio del conjunto de elementos del Dublin Core (DC). Contiene los metadatos DC de los artículos publicados en nueve revistas del área y, a corto plazo, tiene previsto incluir el resto de títulos editados en España.

\section{http://temaria.net}

\section{http://temaria.net/revistas.php}

Tras una primera fase de alimentar el sistema, entre los objetivos propuestos para el año 2006 figura la explotación y la difusión de sus contenidos. Para ello se realizarán nuevas aplicaciones, entre las cuales cabe señalar la difusión mediante RSS de los contenidos indizados.

\subsection{Descripción técnica}

El engranaje de Temaria es parametrizable, y esto marca una diferencia con los mecanismos generadores de fuentes RSS mencionados

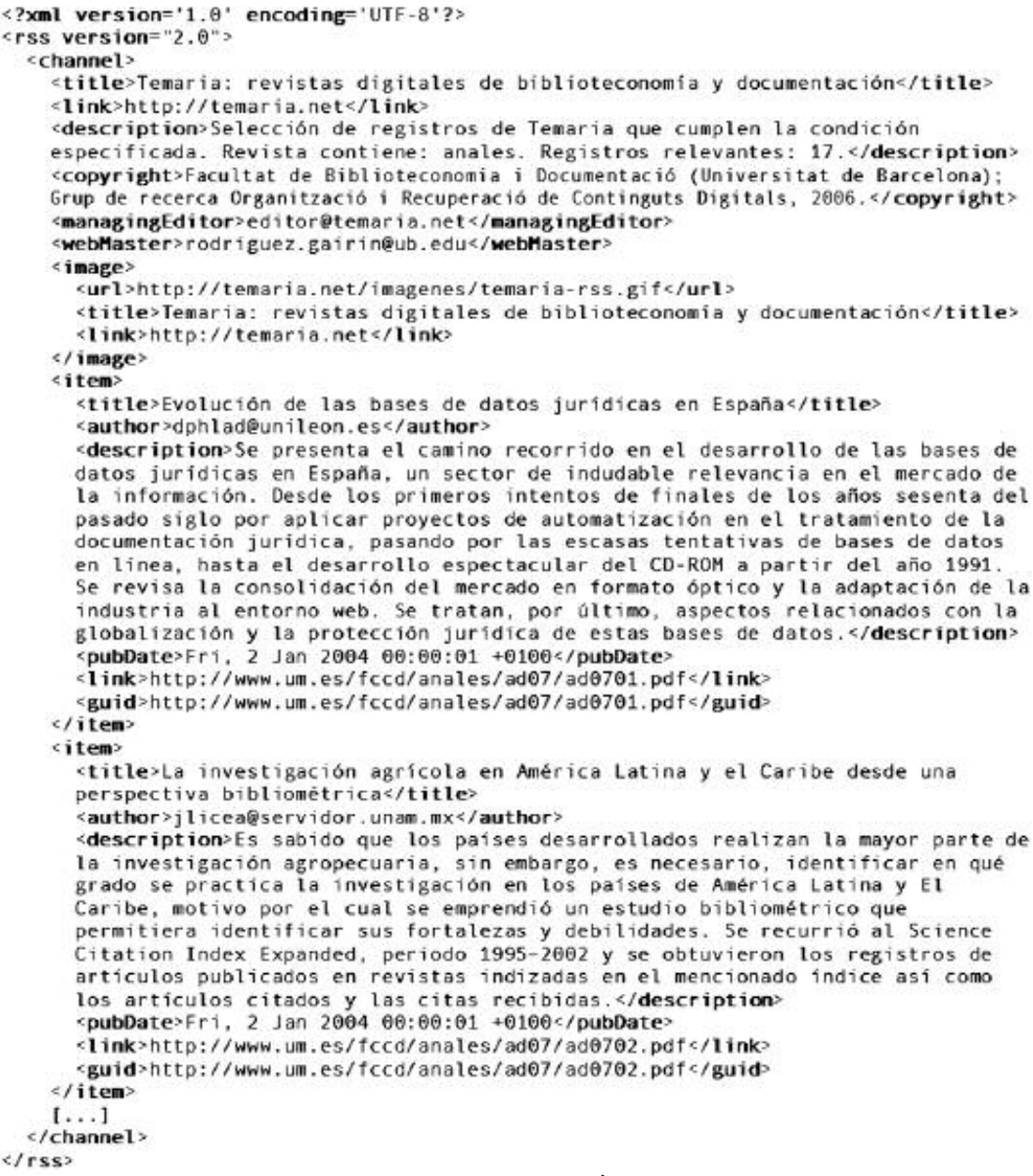

Figura 6. Fuente RSS generada por Temaria

hasta ahora. En efecto, genera los documentos RSS de manera dinámica mediante una interrogación a la base de metadatos DC. Esto amplía la capacidad de sindicación porque proporciona una versatilidad mayor que la que conceden los habituales ficheros estáticos, que son de utilidad limitada.

\section{«Aunque todavía escasas, son varias las aplicaciones de la sindicación al ámbito de la biblioteconomía y documentación»}

Y dado que los artículos se han indizado mediante un vocabulario controlado que señala relaciones semánticas entre sus términos, las consultas por materia amplían la búsqueda de cualquier término a todos los conceptos más específicos, cuando los haya. Por ejemplo, la suscripción a artículos sobre biblio- metría recupera también bibliografía sobre análisis bibliométrico, comunicación científica, indicadores bibliométricos y otras materias relacionadas, igualmente específicas.

\subsection{Posibilidades}

La sindicación de contenidos de Temaria resulta útil para usuarios de diferente índole. Como suscriptores pueden beneficiarse los usuarios finales del portal; y como difusores de información, los editores de las revistas indizadas y los administradores de webs que manejen gestores de contenidos capaces de beber de las fuentes RSS. Véanse otras posibilidades:

-Obtención del sumario del último número de una revista.

Sintaxis: http://temaria.net/ metadatos.php? format $=r s s \& i s s n=$ nnnn-nnnn\&actual $=s$

Porque se trata de un url estático, que actualiza sus contenidos au- 
tomáticamente, el editor de cualquiera de las revistas que se vacían en Temaria puede colocar un botón hipertextual en su sede web y sindicar así el contenido de su publicación sin necesidad de incorporar más tecnología (figuras 2 y 3 ).

\section{«La sindicación de Temaria es \\ parametrizable $y$, en \\ consecuencia, más versátil que las habituales fuentes RSS estáticas, de utilidad limitada»}

\section{- Seguimiento de un autor.}

Sintaxis: http://temaria.net/ metadatos.php? format $=r s s \&$ aulast=autor

Dado que el sistema muestra en primer lugar los artículos más recientes, el agregador detecta la incorporación de nuevos documentos del autor que se especifique (figura 4).

- Seguimiento de un tema.

Sintaxis: http://temaria.net/ metadatos.php? format $=r s s \&$ subject=término

En este caso el sistema busca en el tesauro el término indicado y los conceptos más específicos (expansión) y devuelve el resultado de la consulta (figura 5). http://temaria.net/tesauro.php

—Otras posibilidades.

El sistema de sindicación sigue el estándar OpenURL; por lo tanto es posible formular consultas concretas con los parámetros <title> (título de la revista), <atitle $>$ (título del artículo), <issn>, <date $>$ (fecha de publicación), <issue $>$ (número) y <pages $>$ (paginación).

Ejemplo: artículos publicados en Anales de documentación en 2004 (figura 6): http://temaria.net/ metadatos.php? format $=r s s \&$ title $=$ anale $\&$ date $=2004$

\section{Comentarios}

Es evidente, entonces, que la sindicación facilita la difusión selectiva de la información a los usuarios de servicios web y que ayuda a disminuir la dispersión de las sedes web que son del interés de un usuario determinado; contribuye también a eludir la diversidad de frecuencias en la actualización de los contenidos. En este sentido, existe consenso pleno para considerar que hay numerosas aplicaciones y que son muchas las posibilidades para el ámbito de la biblioteconomía y documentación, pero que todavía no se utilizan ni se explotan de forma masiva.

\section{«El modelo de sindicación de Temaria selecciona la información que responde a intereses determinados y permite difundir contenidos orientados a públicos concretos»}

Incluir prestaciones de sindicación en un portal de revistas, como el caso de Temaria, proporciona la suscripción a una o más publicaciones, que es la finalidad fundamental de este instrumento. A cualquier usuario le permite hacer un seguimiento exhaustivo de los artículos nuevos de un autor o una materia.

Otro beneficio de la sindicación: gracias a su capacidad para difundir contenidos de forma selectiva a partir de un perfil de búsqueda, Temaria puede proporcionar a una sede web el valor añadido que aportan las empresas especializadas en la generación de contenidos. Este modelo parametrizable de sindicación posibilita integrar en un sitio algo más que un sumario de revista: selecciona la información que res- ponde a intereses determinados y permite distribuir contenidos orientados a públicos concretos.

\section{Bibliografía}

Franganillo, Jorge; Catalán, Marcos A. "Bitácoras y sindicación de contenidos: dos herramientas para difundir información”. En: BiD: textos universitaris de biblioteconomia $i$ documentació, 2005, diciembre, n. 15. http://www.ub.edu/bid/15frang2.htm

Moffat, Malcolm. "RSS: a primer for publishers and content providers". En: The new review of information networking, 2003, pp. 123-144.

Pilgrim, Mark. "What is RSS". En: Xml.com, 2002, diciembre.

http://xml.com/lpt/a/2002/12/18/dive-into-xml.html

Portela, Paloma. "Portales, sindicación, contenidos: nuevas oportunidades para los gestores de información”. En: El profesional de la información, 2001, diciembre, v. 10, n. 12, pp. 14-16.

Silva, Alberto. "RSS: informarse sin navegar". En: Consumer, 2004, abril. http://www.consumer.es/web/es/tecnologia/inter net/2004/04/01/98000.php

Serrano, Jorge. "Google, DSI y la sindicación de contenidos mediante RDF/RSS". En: El profesional de la información, 2004, enero-febrero, v. 13 , n. 1, pp. 67-70.

Wusteman, Judith. "RSS: the latest feed". En: Library hi tech, 2004, v. 22, n. 4, pp. 404-413.

\section{Notas}

1. Para más información, véase The Atom publishing protocol.

http://ietfreport.isoc.org/idref/draft-ietf-atompu b-format

2. Existen varios directorios de canales de noticias RSS en español:

Canal RSS.

http://canalrss.com

NoticiasRSS.

http://www.noticiasrss.com

Uats'ap.

http://uatsap.com

3. El repertorio se puede consultar en: http://www.public.iastate.edu/ CYBERSTACKS/ eFeeds.htm

\section{Josep Manuel Rodríguez Gairín,} Jorge Franganillo, Ernest Abadal, Assumpció Estivill, Jesús Gascón. Grupo de investigación Organización y Recuperación de Contenidos Digitales, Departament de Biblioteconomia $i$ Documentació. Universitat de Barcelona.

rodriguez.gairin@ub.edu

franganillo@ub.edu

abadal@ub.edu

estivill@ub.edu

gascon@ub.edu 